\title{
Uterine Corpus Leiomyosarcoma and Endometrial Stromal Sarcoma pNO(i+) TNM Finding v8
}

National Cancer Institute

\section{Source}

National Cancer Institute. Uterine Corpus Leiomyosarcoma and Endometrial Stromal

Sarcoma pNO(i+) TNM Finding v8. NCI Thesaurus. Code C139840.

Uterine corpus leiomyosarcoma or endometrial stromal sarcoma with isolated tumor

cells in regional lymph node(s) no greater than $0.2 \mathrm{~mm}$. (from AJCC 8th Ed.) 